\title{
Progressive sudomotor dysfunction in amyotrophic lateral sclerosis
}

\author{
M Beck, R Giess, T Magnus, I Puls, K Reiners, K V Toyka, M Naumann
}

J Neurol Neurosurg Psychiatry 2002;73:68-70

Autonomic dysregulation is part of the complex degenerative process in amyotrophic lateral sclerosis (ALS). To investigate this, sweating was examined at rest in 39 patients with ALS in comparison with a control group. Sweat was collected over a 30 second period over the thenar and hypothenar eminences and on the sole of the foot, using a commercial device based on vapour pressure gradient. The measurements were repeated after three and six months in 10 patients for longitudinal analysis. In early ALS, patients had significantly higher skin water loss than control subjects over the thenar and the hypothenar eminences. In advanced disease stages, sweating was decreased at all sites compared with controls. A significant decline in sweat secretion of about $40 \%$ was found over a six month period. The findings suggest an abnormal sympathetic activity with hyperhidrosis in early ALS and a reduction in sweat production as the disease progresses.

$\mathrm{T}$ here are recent reports of dysfunction of parasympathetic and sympathetic autonomic innervation in amyotrophic lateral sclerosis (ALS). ${ }^{1}$ Decreased heart rate variation, ${ }^{2}$ alterations of the excretory function of the salivary glands, ${ }^{3}$ and disturbance of the gastrointestinal tract $^{45}$ suggest parasympathetic abnormalities, while studies using ${ }^{123} \mathrm{I}-$ MIBG-SPECT have shown disordered cardiac sympathetic innervation. ${ }^{6}$.

Electrophysiological data have shown alterations of the sympathetic skin responses in ALS, indicating a degeneration of sympathetic nerve fibres, possibly involving sudomotor function. ${ }^{78}$

Postmortem histology shows neuronal degeneration in Onuf's nucleus in the ventral horns of the spinal cord, indicating alterations in bowel and bladder innervation. ${ }^{9}{ }^{10}$ Although autonomic symptoms in ALS are usually subclinical, these findings suggest that ALS may be a multisystem degenerative disorder.

Our aim in this study was to make a quantitative analysis of sudomotor regulation in ALS patients at different stages of their disease and to see whether there was evidence of a progressive deterioration in function.

\section{METHODS}

Thirty nine patients with probable or definite ALS according to the El Escorial criteria ${ }^{11}$ (mean (SD) age 55 (8) years, male to female ratio 0.77, disease duration 20 (8.8) months) and 39 healthy control subjects of comparable age (52 (16) years) and sex ratio (0.92) participated in the study. Their disability was assessed by the ALS functional rating scale (ALSFRS) according to criteria published in $1996 .{ }^{12}$ We measured quantitatively sudomotor function (sweating rate) at three different sites (thenar and hypothenar eminences and the medial sole of the foot), using a commercial device based on the vapour pressure gradient (Evaporimeter, Servo Med AB, Kinna, Sweden). All measurements were done at the same time of day, two hours after the last meal, in a sound insulated, temperature stabilised examination room, following a detailed protocol. This included a 20 minute rest period in the half supine position with eyes closed for adaptation to the ambient temperature (patients, $25.2(0.9)^{\circ} \mathrm{C}$; controls, $25.1(1.0)^{\circ} \mathrm{C}$ ). Any drugs with sympathetic or parasympathetic activity were discontinued at least three days before the measurements. No foods were permitted that might contain compounds known to cause autonomic overactivity (for example caffeine or nicotine).

Data sampling began two minutes after the probe had been positioned. The data represent mean values of water loss measured every two seconds over a 30 second period. Results were automatically extrapolated and shown in $\mathrm{g} / \mathrm{m}^{2} / \mathrm{h}$. The reproducibility of the analysis was tested by repeated measurements on three consecutive days in six patients. An overall coefficient of variation of $15 \%$ was found.

Data on 23 patients with early ALS (mean duration 13.6 (7.6) months, mean ALSFRS 32.6) and 16 heavily disabled patients (mean duration 34.1 (24) months, mean ALSFRS 22.4) were compared with those of a well matched control group. To examine dynamic changes with disease progression, 10 patients were followed up over a six month period.

\section{Statistics}

The effects of sex and age on the measurements in ALS and controls groups were examined by analysis of variance. Differences in the results between ALS patients and controls were determined using Student's $t$ test, after confirmation that the data were normally distributed. Probability $(p)$ values were considered significant at $\mathrm{p}<0.05$.

\section{RESULTS}

By analysis of variance there was no significant influence of sex or age on sweat loss from the thenar and hypothenar eminences, but these variables did affect sweat loss from the sole of foot in the control subjects and in ALS patients in the early stages of the disease. The lowest sweating rates were found on the sole of the foot and the highest on the hypothenar eminence (table 1). This relation remained stable irrespective of disease stage and throughout the longitudinal study. Intraindividual reproducibility was good: the six patients with three sequential measurements on three successive days showed an overall coefficient of variation of $15 \%$.

In early ALS (mean (SD) disease duration 13.6 (7.6) months), the patients had a higher sweat rate $(S)$ than the controls. A significant difference from controls was found at the thenar eminence $\left(\left[\mathrm{S}_{\text {thenar }}\right]_{\mathrm{ALS}}=56.2(19.2) \mathrm{g} / \mathrm{m}^{2} / \mathrm{h} \mathrm{v}\right.$ $\left.\left[\mathrm{S}_{\text {thenar }}\right]_{\text {controls }}=45.5(14.8) \mathrm{g} / \mathrm{m}^{2} / \mathrm{h}, \mathrm{p}<0.05\right)$ and at the hypothenar eminence $\left(\left[\mathrm{S}_{\text {hypothenar }}\right]_{\text {ALS }}=70.9(23.3) \mathrm{g} / \mathrm{m}^{2} / \mathrm{h} v\right.$ $\left.\left[\mathrm{S}_{\text {hypothenar }}\right]_{\text {controls }}=54.1(13.5) \mathrm{g} / \mathrm{m}^{2} / \mathrm{h} ; \mathrm{p}<0.01\right)$. No significant differences could be shown on the sole of foot. In late ALS a lower sweat secretion was found at all three different 
Table 1 Results of quantitative sudomotor analysis

\begin{tabular}{|c|c|c|c|c|c|}
\hline & \multirow[b]{2}{*}{$\mathrm{n}$} & \multicolumn{4}{|c|}{ Sweat loss $\left(\mathrm{g} / \mathrm{m}^{2} / \mathrm{h}\right)$} \\
\hline & & Total & Thenar & Hypothenar & Sole of foot \\
\hline Early stage & 23 & $51.1(26.3)$ & $56.2(19.2)$ & $70.9(23.3)$ & $26.2(11.2)$ \\
\hline Control & 23 & $43.0(20.6)$ & 45.5 (14.8) & $54.1(13.5)$ & $29.3(23.5)$ \\
\hline Late stage & 16 & 27.1 (12.0) & $27.5(5.3)$ & $37.3(9.0)$ & $16.4(10.1)$ \\
\hline Control & 16 & 44.6 (19.8) & 46.0 (12.3) & $54.2(11.6)$ & 33.5 (25.3) \\
\hline
\end{tabular}

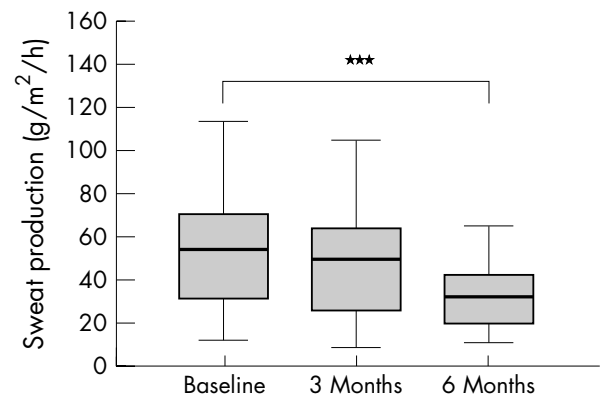

Figure 1 Progressive sudomotor dysfunction over a six month period $(* * * p<0.001$ ). Measurements were performed in 10 equally disabled patients with amyotrophic lateral sclerosis and repeated twice at three month intervals over a follow up period of six months. The data represent mean values of water loss recorded at a frequency of $0.5 \mathrm{~Hz}$ over 30 seconds. Results were automatically extrapolated and are shown in $\mathrm{g} / \mathrm{m}^{2} / \mathrm{h}$. Mean values (horizontal bars) are shown, with range (grey area) and 2SD (vertical bars).

measurement sites compared with the controls: $\left[\mathrm{S}_{\text {thenar }}\right]_{\mathrm{ALS}}=$ $27.5 \quad(5.3) \mathrm{g} / \mathrm{m}^{2} / \mathrm{h} \quad v \quad\left[\mathrm{~S}_{\text {thenar }}\right]_{\text {controls }}=46.0 \quad(12.3) \mathrm{g} / \mathrm{m}^{2} / \mathrm{h}$; $\mathrm{p}<0.001$; $\left[\mathrm{S}_{\text {hypothenar }}\right]_{\text {ALS }}=37.3(9.0) \mathrm{g} / \mathrm{m}^{2} \mathrm{~h} v\left[\mathrm{~S}_{\text {hypothenar }}\right]_{\text {controls }}=$ $54.2(11.6) \mathrm{g} / \mathrm{m}^{2} / \mathrm{h}, \mathrm{p}<0.001 ;\left[\mathrm{S}_{\text {foot }}\right]_{\text {ALS }}=16.4(10.1) \mathrm{g} / \mathrm{m}^{2} / \mathrm{h} v$ $\left[\mathrm{S}_{\text {foot }}\right]_{\text {controls }}=33.6(25.3) \mathrm{g} / \mathrm{m}^{2} / \mathrm{h} ; \mathrm{p}<0.05$.

A follow up evaluation in a subgroup of 10 similarly disabled patients showed a significant decrease of about $40 \%$ in sweat production rate over six months $(p<0.001)$, indicating progressive loss of sudomotor function with disease progression (fig 1).

\section{DISCUSSION}

In the early stages of ALS, we found increased sweating on the palm and reduced sweating on the sole of the foot. In advanced stages of the disease, sweating was reduced at both sites. In longitudinal studies, progression of the autonomic disturbance paralleled motor dysfunction with advancing disease.

With our protocol, we avoided the measurement bias that may be introduced by various factors influencing autonomic function. Room temperature, body temperature, activity, diseases such as thyroid disorders, emotional stress, certain drugs, and smoking are all important factors that can affect autonomic activity. We found that assessment of sweat rate using vapour pressure gradient analysis was a safe and reliable technique. Secondary factors such as immobilisation and muscular atrophy that could affect sweat production were unavoidable but more than one recording site was chosen to diminish bias.

Higher sweat rates shortly after disease onset may indicate denervation hypersensitivity of the sweat glands or a higher autonomous neural firing rate. Chida et al reported augmented sympathetic and decreased parasympathetic function in early ALS, ${ }^{13}$ while Shindo et al found a higher sympathetic firing rate in microneurographic measurements of muscle sympathetic nerve activity in mildly disabled ALS patients compared with other neuromuscular disorders ${ }^{7}$; their patients had only mild to moderate bulbar signs and were able to walk unaided. Thus those results are in keeping with our finding of an increased sweat rate in mildly affected patients, indicating higher sympathetic activity in the early disease stages, and a reduced sweat rate in advanced disease, suggesting a progressive deterioration of the sudomotor system. Degeneration of postganglionic parasympathetic fibres may cause also atrophy of the sweat glands and resulting hypohidrosis. These findings are in line with earlier electrophysiological studies showing abnormal sympathetic skin responses in late ALS, which indicated postganglionic sympathetic dysfunction affecting epidermal and dermal structures. ${ }^{8}{ }^{14}$ They are also in line with the results of pathological studies indicating involvement of the peripheral sensory and autonomic nervous system. ${ }^{15-17}$

Impaired sweating occurs in other peripheral and central neurodegenerative diseases ${ }^{18-21}$ - for example, in multiple system atrophy and progressive autonomic failure, both postganglionic and preganglionic degeneration has been shown to be responsible for hypohidrosis. ${ }^{22}$ In hereditary sensory autonomic neuropathy, postganglionic degeneration has been shown by sympathetic skin response studies. However, published reports on increased sweating at early disease stages are lacking.

While autonomic dysfunction does not play a major clinical role in patients suffering from ALS, its presence supports the view that ALS is a multisystem disorder. Patients often report increased or reduced sweating of their hands or feet, reduced skin temperature, or skin discoloration. Skin biopsies have demonstrated abnormal collagen texture and atrophy of subepidermal structures including the sweat glands. ${ }^{23}$ Small blood vessels show ultrastructural changes such as duplication of the basal membranes and deposition of $\beta$ amyloid. ${ }^{24}$ Moreover, electrophysiological and histopathological changes in the sensory system ${ }^{25}$ indicate a multisystem disorder. We did not perform skin biopsies to demonstrate loss of terminal autonomic nerve fibres directly, because this would have been too invasive. It is thus uncertain how much of the progressive hypohidrosis is caused by structural damage and how much by loss of function in existing nerve fibres.

\section{Authors' affiliations}

M Beck, R Giess, T Magnus, I Puls, K Reiners, K V Toyka,

M Naumann, Department of Neurology, Julius-Maximilians University, Wuerzburg, Germany

Correspondence to: Dr Marcus Beck, Department of Neurology, Julius-Maximilians University, Josef-Schneider-Strasse 11, 97080

Wuerzburg, Germany; marcus.beck@mail.uni-wuerzburg.de

Received 11 May 2001

In revised form 7 March 2002

Accepted 7 March 2002

\section{REFERENCES}

1 Pinelli $\mathbf{P}$, Pisano F, Miscio G. The possible role of a secondary pathogenetic factor in amyotrophic lateral sclerosis. Adv Neurol 1995;68:29-40. 
2 Pisano F, Miscio G, Mazzero G et al. Decreased heart rate variability in amyotrophic lateral sclerosis. Muscle Nerve 1995;18:1225-31.

3 Giess R, Naumann M, Werner E, et al. Injections of botulinum toxin A into the salivary glands improve sialorrhoea in amyotrophic lateral sclerosis. J Neurol Neurosurg Psychiatry 2000;69:121-3.

4 Toepfer M, Folwaczny C, Lochmüller $\mathrm{H}$, et al. Noninvasive (13)C-octanoic acid breath test shows delayed gastric emptying in patients with amyotrophic lateral sclerosis. Digestion 1999;60:567-71.

5 Toepfer M, Schroeder M, Klauser A, et al. Delayed colonic transit times in amyotrophic lateral sclerosis assessed with radio-opaque markers. Eur J Med Res 1997:2:473-6.

6 Druschky A, Spitzer A, Platsch G, et al. Cardiac sympathetic denervation in early stages of amyotrophic lateral sclerosis demonstrated by ${ }^{123}$ I-MIBG-Spect. Acta Neurol Scand 1999;99:308-14.

7 Shindo K, Tsunoda S, Shiozawa Z. Increased sympathetic oufflow to muscles in patients with amyotrophic lateral sclerosis: a comparison with other neuromuscular patients. J Neurol Sci 1995;134:57-60.

8 Masur H, Schulte-Oversohl U, Papke K, et al. Sympathetic skin response in patients with amyotrophic lateral sclerosis. Funct Neurol

1995; 10:131-5

9 Pullen $\mathbf{A H}$, Martin JE. Ultrastructural abnormalities with inclusions in Onuf's nucleus in motor neuron disease (amyotrophic lateral sclerosis). Neuropathol Appl Neurobiol 1995;21:327-40.

10 Carvalho M, Schwartz MS, Swash M. Involvement of the external anal sphincter in amyotrophic lateral sclerosis. Muscle Nerve 1995; 18:848-53.

11 World Federation of Neurology. Research Group on Neuromuscular Diseases, El Escorial. World Federation of Neurology criteria for the diagnosis of amyotrophic lateral sclerosis. J Neurol Sci 1994; 124(suppl):96-107.

12 ACTS Study Group. The amyotrophic lateral sclerosis functional rating scale. Assessment of activities of daily living in patients with amyotrophic lateral sclerosis. The ALS CNTF treatment study (ACTS) phase I-II Study Group. Arch Neurol 1996;53:141-7.
13 Chida K, Sakamaki S, Takasu T. Alteration in autonomic function and cardiovascular regulation in amyotrophic lateral sclerosis. J Neurol 989;236: 127-30.

14 Dettmers C, Fatepour D, Faust $\mathrm{H}$, et al. Sympathetic skin response abnormalities in amyotrophic lateral sclerosis. Muscle Nerve 1993; 16:930-4.

15 Bradley WG, Good P, Rasool CG, et al. Morphometric and biochemical studies of peripheral nerves in amyotrophic lateral sclerosis. Arch Neurol 1983:41:267-77.

16 Dyck PJ, Stevens JC, Mulder DW. Frequency of nerve fiber degeneration of peripheral motor and sensory neurons in amyotrophic lateral sclerosis. Neurology (Minneapolis) 1975;25:781-5.

17 Steiner TJ, Sethi KD, Rose FC. Autonomic function in motor neurone disease. In: Rose FC, ed. Research progress in motor neurone disease. London: Pitman, 1984:180-8.

18 Hilz MJ, Stemper B, Axelrod FB. Sympathetic skin response differentiates hereditary sensory autonomic neuropathies III and IV. Neurology 1999.52:1652-7.

19 Chokroverty S. Autonomic dysfunction in olivopontocerebellar atrophy. Adv Neurol 1984;41:105-41.

20 Robinson B, Johnson R, Abernethy D, et al. Familial distal dysautonomia. J Neurol Neurosurg Psychiatry 1989:52:1281-5.

21 Wolfe GI, Galetta SL, Teener JW, et al. Site of autonomic dysfunction with Ross's syndrome and postganglionic Horner's syndrome. Neurology 1995:45:2094-6.

22 Benarroch EE, Smithson IL, Low PA, et al. Depletion of catecholaminergic neurons of the rostral ventrolateral medulla in multiple system atrophy with autonomic failure. Ann Neurol 1998;43:149-51.

23 Provinciali L, Cangiotti A, Tulli D, et al. Skin abnormalities and autonomic involvement in the early stage of amyotrophic lateral sclerosis. J Neurol Sci 1994:126:54-61.

24 Kolde G, Bachus R, Ludolph AC. Skin involvement in amyotrophic lateral sclerosis. Lancet 1996;347:1226-7.

25 Theys PA, Peeters E, Robberecht W. Evolution of motor and sensory deficits in amyotrophic lateral sclerosis estimated by neurophysiological techniques. J Neurol 1999;246:438-42. 